\title{
On the instanton-induced portion of the nucleon strangeness
}

\author{
D. Klabučar ${ }^{1 a}$, K. Kumerički ${ }^{1 b}$, B. Melić ${ }^{2 c}$ and I. Picek ${ }^{1 d}$ \\ ${ }^{1}$ Department of Physics, Faculty of Science, University of Zagreb, POB 162, HR-10001 Zagreb, Croatia \\ 2 Theoretical Physics Division, Ruđer Bošković Institute, Bijenička cesta 54, HR-10001 Zagreb, Croatia
}

\begin{abstract}
We calculate the instanton contribution to the proton strangeness in the MIT bag enriched by the presence of a dilute instanton liquid. The evaluation is based on expressing the nucleon matrix elements of bilinear strange quark operators in terms of a model valence nucleon state and interactions producing quark-antiquark fluctuations on top of that valence state. Our method combines the usage of the evolution operator containing a strangeness source, and the Feynman-Hellmann theorem. It enables one to evaluate the strangeness in different Lorentz channels in, essentially, the same way. Only the scalar channel is found to be affected by the interaction induced by the random instanton liquid.
\end{abstract}

\section{Introduction}

Despite the accumulated evidence for the nucleon strangeness, there has been as yet no balanced understanding of its various appearances. By a particular nucleon strangeness we understand the value of the nucleon matrix element $\left\langle N\left|\mathcal{O}_{s}(\Gamma)\right| N\right\rangle$, where the bilinear $\mathcal{O}_{s}(\Gamma)=$ $\bar{s} \Gamma s$ might represent the scalar, pseudoscalar, vector, axial vector and tensor strange current densities $(\Gamma=$ $\left.1, \gamma_{5}, \gamma_{\mu}, \gamma_{\mu} \gamma_{5}, \sigma_{\mu \nu}\right)$. Thus, any interaction $\mathcal{L}_{I}$ that induces $s \bar{s}$ pairs in the nucleon state $|N\rangle$ potentially leads to various types of nucleon strangeness. The imaginable interactions $\mathcal{L}_{I}$, which are related to QCD-vacuum fluctuations, might prefer some of the strangeness channels. In particular, there is a conjecture [1] that a nontrivial QCD-vacuum structure selects the pseudoscalar and scalar channels, which experience the axial and trace anomaly, respectively. In the present paper we focus on QCD-vacuum fluctuations as given by the instantonliquid model 2, 3, 团, i. e. we take $\mathcal{L}_{I} \rightarrow \mathcal{L}_{\text {inst }}$. Such an interaction generates an $s$-quark loop (schematically shown in Fig. 11) to which an external probe can couple. It is important that this interaction can be treated perturbatively and enables us to compare its relative contri-

\footnotetext{
aklabucar@phy.hr

bkkumer@phy.hr

${ }^{\mathrm{c}}$ melic@thphys.irb.hr

d picek@phy.hr
}

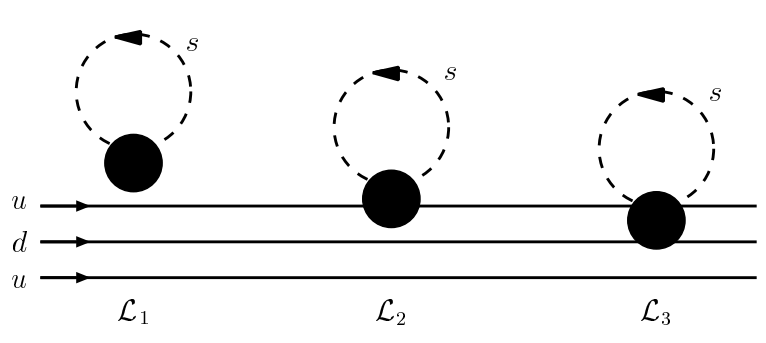

Figure 1: Instanton-induced local strangeness represented by the effective one-, two- and three-body operators. Non-strange quarks are denoted by solid lines, and strange ones by dashed lines.

butions to different strangeness channels. The relatively complicated interaction $\mathcal{L}_{I}=\mathcal{L}_{\text {inst }}$ [given by Eqs. (22)(24) below] is conveniently split into three pieces:

$$
\mathcal{L}_{I}=\mathcal{L}_{1}+\mathcal{L}_{2}+\mathcal{L}_{3}
$$

where the parts illustrated in Fig. 11 refer to the one-, two- and three-body operators. These operators change the known valence (model) state $\left|N_{0}\right\rangle$ to the state $|N\rangle$ containing the $s \bar{s}$ pairs. Then, we provide an expression (Eq. (19)) suitable for computing the strange matrix element of the full nucleon state, $\langle N|: \bar{s} \Gamma s:| N\rangle$.

The current evidence for the strangeness content of the proton comes from the external probe both at lowand at high-momentum transfers. The analysis of the term $\sigma_{\pi N}$ in low-energy $\pi N$ scattering reveals comparable light and strange quark nucleon matrix elements [1] ( $N$ means the proton throughout this paper):

$$
\begin{aligned}
\langle N|\bar{u} u| N\rangle & \simeq 4.8, \\
\langle N|\bar{d} d| N\rangle & \simeq 4.1, \\
\langle N|\bar{s} s| N\rangle & \simeq 2.8,
\end{aligned}
$$

i.e., the unexpectedly large scalar strangeness. A posteriori, it is found to be in accordance with QCD-vacuum characteristics [1], as represented, for example, by the (naive) bag-model relation [5]

$$
\langle N|\bar{s} s| N\rangle=-\langle 0|\bar{s} s| 0\rangle V
$$

or the QCD sum-rules result [6]

$$
\langle N|\bar{s} s| N\rangle \simeq 2.4 \text {. }
$$




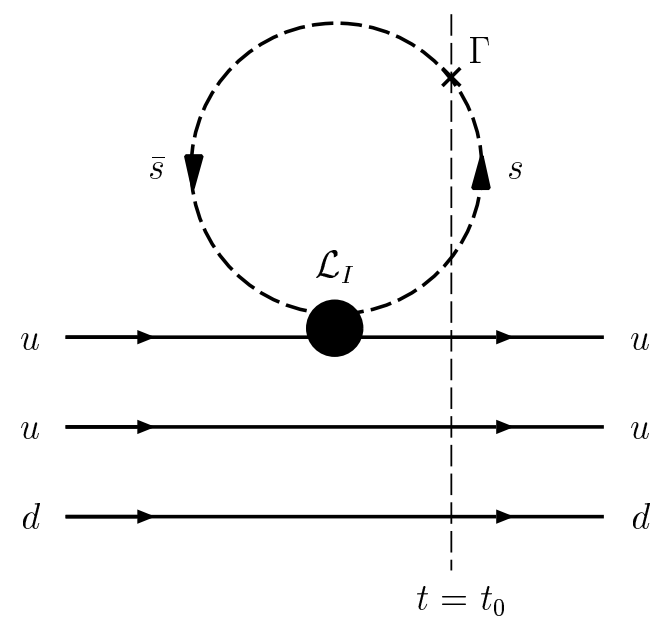

Figure 2: Non-vanishing nucleon strangeness due to a response of the valence nucleon state to a strangeness source at $\Gamma($ denoted by $\times)$, i.e. to a probe coupled to strange quarks through $\Gamma$. More precisely, this graph is that part of the nucleon response which arises only through one interaction $\mathcal{L}_{I}$.

The other piece of evidence for the strangeness content of the proton comes from the polarized leptonnucleon scattering at relatively high-momentum transfer, higher than the scales pertinent for our considerations. The analysis [7] of new data supports the original EMC findings [8, 9 - it reveals a non-vanishing fraction $\Delta s=-0.11 \pm 0.06$ of the proton spin $S_{\mu}$ carried by the $s$ quark. This is not negligible in comparison with $\Delta u=0.82 \pm 0.06$ and $\Delta d=-0.44 \pm 0.06 . \Delta s$ is related to the axial strangeness of the proton defined as $\left\langle N\left|\bar{s} \gamma_{\mu} \gamma_{5} s\right| N\right\rangle=\Delta s S_{\mu}$.

The vector strangeness, described by the Dirac and the Pauli form factors as

$\left\langle N\left|\bar{s} \gamma_{\mu} s\right| N\right\rangle=\bar{u}_{N}\left(p^{\prime}\right)\left[F_{1}^{s}\left(q^{2}\right) \gamma_{\mu}+F_{2}^{s}\left(q^{2}\right) \frac{i \sigma_{\mu \nu} q^{\nu}}{2 M_{N}}\right] u_{N}(p)$,

can be related to the analogous flavour singlet (0) and the hypercharge (8) form factors for $\left\langle N\left|V_{\mu}^{(0,8)}\right| N\right\rangle$ through

$$
\begin{aligned}
\bar{s} \gamma_{\mu} s & =V_{\mu}^{(0)}-2 V_{\mu}^{(8)}, \\
V_{\mu}^{(0)} & =\frac{1}{3} \bar{q} \gamma_{\mu} q, \quad V_{\mu}^{(8)}=\frac{1}{\sqrt{3}} \bar{q} \gamma_{\mu} \frac{\lambda^{8}}{2} q .
\end{aligned}
$$

Although $F_{1}^{s}(0)=0$ as the net nucleon strangeness, its momentum dependence determines the strangeness radius

$$
r_{s}^{2}=\left.6 \frac{d}{d q^{2}} F_{1}^{s}\left(q^{2}\right)\right|_{q^{2}=0},
$$

while the strange magnetic moment is given by

$$
\mu_{s}=F_{2}^{s}(0) .
$$

Note the relation $F_{2}^{s}=F_{2}^{(0)}-\frac{2}{\sqrt{3}} F_{2}^{(8)}$, where the last term is constrained by $\frac{2}{\sqrt{3}} F_{2}^{(8)}=\kappa_{p}+\kappa_{n}=-0.12$. Knowledge of the $F_{2}^{(0)}$ flavour singlet term would also enable one to determine the baryomagnetic moment

$$
\mu_{p}^{(0)}=F_{1}^{(0)}(0)+F_{2}^{(0)}(0)=\left(1+F_{2}^{(0)}\right) \text { n. m. }
$$

There are many various model-dependent calculations $[10,11,12,13,14,15,16,17,18,19,20,21,22$ of the quantities listed above. By deriving Eqs. (19)(20) in the next section, we provide a framework which is rather general in that it can be applied to different quark models and flavor-mixing interactions $\mathcal{L}_{I}$. We illustrate its usage on the example of the MIT bag model and instanton-induced interaction. At least in principle, this framework also treats all Lorentz channels on an equal footing, depending on which $\Gamma$ is plugged in Eqs. (19) $-(20)$.

\section{Nucleon strangeness induced on top of the valence quark state}

It is not very surprising in non-perturbative QCD, in the light of its non-vanishing quark scalar condensates, that some matrix elements $\langle N|\bar{s} \Gamma s| N\rangle$ can be markedly different from zero. The vacuum expectation value of $\bar{s} s$ is actually approximately as large as for non-strange quarks: $\langle 0|\bar{s} s| 0\rangle \approx\langle 0|\bar{u} u| 0\rangle=\langle 0|\bar{d} d| 0\rangle$, i.e. roughly equal to or even more negative than $(-200 \mathrm{MeV})^{3}$. The MIT bag model provides a good illustration how this leads to a large $\langle N|\bar{s} s| N\rangle$ [5]. However, there may also be $s \bar{s}$-pairs other than those from the QCD-vacuum condensate, so that normal-ordered strange operators can, in principle, also have non-vanishing nucleon matrix elements.

Since we are interested in the $s \bar{s}$-pairs that may exist in addition to those from the (non-perturbative-)vacuum channel, it is convenient to define the normal ordering with respect to the non-perturbative vacuum $|0\rangle$ :

$$
: \bar{q} \Gamma q:=\bar{q} \Gamma q-\langle 0|\bar{q} \Gamma q| 0\rangle
$$

Ideally, this referent vacuum state $|0\rangle$ would be the true non-perturbative vacuum of QCD, but since in this paper we are concerned with quark models imitating QCD, Eq. (10) will in practice mean that the normal ordering is taken with respect to a model vacuum state. By this we mean the ground nucleon state from which the valence quarks are removed (for example, the "empty bag" in the case of the MIT bag model). For the strange quarks, the normal ordering with respect to this referent vacuum state $|0\rangle$ is equivalent to the normal ordering with respect to the model nucleon ground state $\left|N_{0}\right\rangle$ composed of the non-strange valence quarks only. Of course, such a definition of normal ordering is then necessarily 
tied to the characteristic hadronic scale of $\sim 1 \mathrm{GeV}$, at which the non-perturbative QCD effects dominate and at which, and below which, quark models provide a reasonable description of the nucleon bound state.

Figure 2 illustrates how a non-vanishing value not only of $\langle N|\bar{s} \Gamma s| N\rangle$, but also of the normal-ordered $\langle N|$ : $\bar{s} \Gamma s:|N\rangle$ can then come about: at the instant $t=t_{0}$ the composite nucleon is hit by an external probe (e.g. a neutrino [23]) with the coupling $\Gamma$ to strange quarks. Owing to an interaction capable of producing $s \bar{s}$ fluctuations, the nucleon state $|N\rangle$ at the time slice $t=t_{0}$ obviously contains not only the valence quarks uud, but also the $s$-quark loop to which the external probe can also couple.

Let us schematically write down the full nucleon (proton) state, which is also coupled to the strangeness-sensitive probe:

$$
\begin{aligned}
|N\rangle & =\frac{1}{\mathcal{N}}\left(\sum_{X=0}^{\infty} C_{X}|u u d X\rangle+\sum_{X=0}^{\infty} C_{s \bar{s} X}|u u d s \bar{s} X\rangle\right) \\
& \equiv \frac{1}{\mathcal{N}}\left(\left|N_{0}\right\rangle+|\delta N\rangle\right) .
\end{aligned}
$$

Here, $X$ (starting from $X=|0\rangle$, the complicated non-perturbative $Q C D$ vacuum) symbolizes any number of various perturbative and non-perturbative gluon and quark configurations including quark-antiquark pairs and, in particular, strange pairs which escaped detection by this probe. These complicated configurations "dress" quarks $(q=u, d, s, \ldots)$ into their effective counterparts - constituent quarks $\mathcal{Q}=\mathcal{U}, \mathcal{D}, \mathcal{S}, \ldots$. In terms of the constituent quarks, this part, unperturbed by the strangeness-sensitive probe, is just the valence part $\left|N_{0}\right\rangle=|\mathcal{U} \mathcal{U D}\rangle$ when the nucleon is a proton. It is obvious in terms of the constituent quarks that $\left\langle N_{0}|: \bar{s} \Gamma s:| N_{0}\right\rangle=0$. The one strange pair detected at $\Gamma$ has been explicitly denoted by $s \bar{s}$ in the $|\delta N\rangle$-part of the nucleon state perturbed by the probe. $|\delta N\rangle$ can be viewed as the response of $\left|N_{0}\right\rangle$ to the weakly coupled strangeness-sensitive probe. The coefficients $C_{X}, C_{s \bar{s} X}$ denote the amplitudes of states with various admixtures $X$ or $s \bar{s} X . \mathcal{N}$ is the normalization. This response makes possible that the total nucleon $\Gamma$-strangeness $\langle N|\bar{s} \Gamma s| N\rangle$ also receives a non-vanishing contribution from the non-vacuum channel $\langle N|: \bar{s} \Gamma s:| N\rangle$.

However, the question is how to get the nucleon state in sufficiently specific terms in order to have a calculable expression for $\langle N|: \bar{s} \Gamma s:| N\rangle$. A viable approach is to resort to a constituent model of hadrons. The idea of a constituent model is that all the mess of fluctuations $X$ is by some model parameterization lumped into dressing of constituent quarks $\mathcal{Q}$, as well as into effective model interactions, or a mean field they feel. The valence proton state $\left|N_{0}\right\rangle$ would then be identified with the model ground eigenstate $|\mathcal{U} \mathcal{U} \mathcal{D}\rangle$ built up only of non-strange effective quarks (so that $\left\langle N_{0}|: \bar{s} \Gamma s:| N_{0}\right\rangle=0$, even though possibly $\left\langle N_{0}|\bar{s} \Gamma s| N_{0}\right\rangle \neq 0$, at least for $\Gamma=1$, owing to the strange vacuum condensate). Let us denote all possible higher eigenstates of some model Hamiltonian $H_{0}$ by $|k\rangle$ :

$$
H_{0}\left|N_{0}\right\rangle=E_{N_{0}}\left|N_{0}\right\rangle, \quad H_{0}|k\rangle=E_{k}|k\rangle, \quad E_{k}>E_{N_{0}} .
$$

The Hamiltonian $H_{0}$ is responsible for the formation of (model) hadron states composed of definite, fixed numbers of quarks. In the simplest case, we can imagine $H_{0}$ as consisting of a sum of one-body quark operators, say typically of the effective quark kinetic energy operator and the mean, or self-consistent, field in which the dressed valence quarks would move. For example, $H_{0}$ could be the static bag-model Hamiltonian. $\left|N_{0}\right\rangle$ would then be the bag-model nucleon in its ground state, and $|k\rangle$ all higher bag states with a definite number of constituents. In any case, $H_{0}$ defines the nucleon model - possibly together with some other ingredients (such as the confining boundary condition in bag models).

What $H_{0}$ cannot do is to produce $s \bar{s}$ fluctuating pairs. To produce such pairs, we have to supplement $H_{0}$ defining the model one starts from, by some Hamiltonian $H_{I}$ (corresponding to the Lagrangian density $\mathcal{L}_{I}$ ) which can produce $s \bar{s}$ excitations on top of $\left|N_{0}\right\rangle$. This means that $\mathcal{L}_{I}$, and thus also $H_{I}$, contains strange quark field operators bilinearly, so that it can connect $\left|N_{0}\right\rangle$ with $|\delta N\rangle$ containing $s \bar{s}$ pairs.

To clarify that introducing $\mathcal{L}_{I}$ does not lead to double-counting, let us repeat that $H_{0}$ is just a model Hamiltonian, the parameters of which should mimic the effects of full, true non-perturbative QCD as much as possible. For example, if $H_{0}$ is the Hamiltonian of the non-relativistic naive constituent quark model, it must contain the postulated mass parameter of the constituent quark mass $M_{\mathcal{Q}} \approx M_{N_{0}} / 3$. The corresponding quantity in the true theory, the dynamically generated quark mass, is (in principle) the result of all possible QCD interactions, so that the interactions related to $H_{I}$ can, in real QCD, also contribute to this mass by contributing to the $s \bar{s}$-fluctuations. The dynamically generated non-strange quark mass must be close to the model constituent quark mass parameter $M_{\mathcal{Q}}$ sitting in $H_{0}$, and only in such implicit, indirect ways are interactions like $H_{I}$ "present" in $H_{0}$. However, they are not present explicitly, and, in fact, $H_{0}$ cannot produce any $s \bar{s}$ fluctuations at all. Therefore, if we want to study the $s \bar{s}$ fluctuations, we must introduce $H_{I}$ to enrich the model nucleon with $\mathcal{S} \overline{\mathcal{S}}$-fluctuations on top of $\left|N_{0}\right\rangle$.

In order to obtain the expression for $\langle N|: \bar{s} \Gamma s:| N\rangle$ by utilizing the Feynman-Hellmann theorem [24, 25], let us define an auxiliary perturbation Hamiltonian $H^{\prime}$ by adding to $H_{I}$ a source term for the strange operator we want to calculate in the "full" nucleon state $|N\rangle$ :

$$
H^{\prime} \equiv H_{I}+\lambda \otimes<\bar{s} \Gamma s>
$$

where $\langle\bar{s} \Gamma s\rangle$ is the convenient abbreviation 


$$
<\bar{s} \Gamma s>\equiv \int \bar{s}(x) \Gamma s(x) d^{3} x .
$$

The generic form $\lambda \otimes \Gamma$ can mean any of the cases $\lambda \mathbf{1}_{4}$, $\lambda_{\mu} \gamma^{\mu}, \lambda_{5 \mu} \gamma^{\mu} \gamma_{5}, \lambda_{\mu \nu} \sigma^{\mu \nu}$, etc.

Then we use the auxiliary perturbation Hamiltonian as the interaction Hamiltonian in the evolution operator $U\left(t_{2}, t_{1}\right)$. The perturbation expansion of this operator is

$$
\begin{aligned}
U\left(t_{2}, t_{1}\right) & =1+\sum_{n=1}^{\infty} U^{(n)}\left(t_{2}, t_{1}\right) \\
& =\hat{T}\left\{1+\sum_{n=1}^{\infty} \frac{i^{n}}{n !}\left[\int_{t_{1}}^{t_{2}}: L_{\text {int }}(t): d t\right]^{n}\right\} .
\end{aligned}
$$

Here, $\hat{T}$ denotes the time-ordering operator and $L_{\text {int }}(t)=$ $\int \mathcal{L}_{\text {int }}(\mathbf{x}, t) d^{3} x=-H_{\text {int }}(t)$ is the interaction Lagrangian to be replaced with the form containing the strangeness sources, as in the definition of $H^{\prime}$ (Eq. (13p):

$$
\begin{aligned}
L(t)_{\mathrm{int}} \rightarrow L^{\prime}(t) & =L_{I}(t)-\lambda \otimes<\bar{s} \Gamma s(t)> \\
& =\int d^{3} x\left[\mathcal{L}_{I}(x)-\lambda \otimes \bar{s}(x) \Gamma s(x)\right](16)
\end{aligned}
$$

The Feynman-Hellmann theorem then enables one to understand the nucleon matrix elements of the strange current densities, $\langle N|: \bar{s} \Gamma s:| N\rangle$, as the response (to the strange current source) of the $\left\langle N_{0}(t \rightarrow+\infty)\right| N_{0}(t \rightarrow$ $-\infty)\rangle$ transition amplitude of the model ground state $\left|N_{0}\right\rangle$. For example, in the case of the second-order term in Eq. (15), the substitution (16) leads to

$$
\begin{aligned}
& U^{(2)}(+\infty,-\infty)=-\frac{1}{2} \hat{T} \int_{-\infty}^{+\infty} d t \int_{-\infty}^{+\infty} d t^{\prime}\left[: L_{I}(t):: L_{I}\left(t^{\prime}\right):\right. \\
& -\lambda_{\alpha}:<\bar{s} \gamma^{\alpha} s(t)>:: L_{I}\left(t^{\prime}\right):-: L_{I}(t): \lambda_{\beta}:<\bar{s} \gamma^{\beta} s\left(t^{\prime}\right)>: \\
& \left.+\lambda_{\alpha} \lambda_{\beta}:<\bar{s} \gamma^{\alpha} s(t)>::<\bar{s} \gamma^{\beta} s\left(t^{\prime}\right)>:\right] .
\end{aligned}
$$

For definiteness, the above expression for $U^{(2)}$ has been written for the vector strange current density. The firstorder contribution to the vector nucleon strangeness can then be obtained by considering

$$
\left.\frac{\partial}{\partial \lambda_{\mu}}\left\langle N_{0}\left|U^{(2)}(+\infty,-\infty)\right| N_{0}\right\rangle\right|_{\lambda_{\mu}=0} .
$$

In general, for any matrix $\Gamma$ in the spinor space, the strange nucleon matrix element of the full nucleon state $|N\rangle$ is, to the two lowest orders (due to the $U^{(2)}$ and $U^{(3)}$ terms), given by

$$
\begin{aligned}
& \langle N|: \bar{s} \Gamma s:| N\rangle=i \int_{-\infty}^{+\infty} d t^{\prime}\left\langle N_{0}\right| \hat{T}:\left\langle\bar{s} \Gamma s\left(t_{0}\right)>:\right. \\
& \times: L_{I}\left(t^{\prime}\right):\left|N_{0}\right\rangle-\frac{1}{2} \int_{-\infty}^{+\infty} d t^{\prime} \int_{-\infty}^{+\infty} d t^{\prime \prime} \\
& \times\left\langle N_{0}\left|\hat{T}:<\bar{s} \Gamma s\left(t_{0}\right)>:: L_{I}\left(t^{\prime}\right):: L_{I}\left(t^{\prime \prime}\right):\right| N_{0}\right\rangle .
\end{aligned}
$$

Obviously, the non-vanishing contributions to (19) occur only when the strange quark fields are fully contracted. For example, the integrand of the first term in (19), written in terms of space integrals over the contracted strange current and Lagrangian densities, is

$$
\begin{aligned}
\int d^{3} x d^{3} x^{\prime}\left\langle N_{0}\left|\hat{T}: \bar{s}(x) \Gamma s(x):: \mathcal{L}_{I}\left(x^{\prime}\right):\right| N_{0}\right\rangle \\
=\int d^{3} x d^{3} x^{\prime}\left\langle N_{0}|: \overbrace{\bar{s}(x) \Gamma \underbrace{s(x) \mathcal{L}_{I}}}\left(x^{\prime}\right):| N_{0}\right\rangle,
\end{aligned}
$$

where the contractions are indicated by over- and underbraces, and $t_{0} \equiv x_{0}$ and $t^{\prime} \equiv x^{\prime}{ }_{0}$, for consistency of the notation. So, the first term in (19) corresponds to Fig. 2, since these contractions, or time-ordered pairings, are, of course, the propagators of strange quarks. In the second term, the two contractions must connect the strangeness source at $\Gamma$ with two different separately normal-ordered interaction Lagrangian densities which act as "sinks" for strangeness at two different points of the valence-quark lines. In any case, there must be an additional strange-quark contraction between these two : $\mathcal{L}_{I}$ :'s, and this completes the strange-quark loop. Fig. 3 shows an example of the graphs originating from the second term of (19), namely the $U^{(3)}$ contribution. Clearly, in this way, one can generate contributions corresponding to kaon-baryon loops in the approaches employing hadron degrees of freedom. Below, we will use a strangeness-generating interaction $\mathcal{L}_{I}$ which is perturbative, so that we do not expect sizable contributions to the $s \bar{s}$-effects from the second order in $\mathcal{L}_{I}$. In addition to that, there are indications that these contributions related to strange meson loops, should be rather small even when one does not restrict oneself to perturbative $s \bar{s}$-generating interactions. Some of these indications come from model-dependent calculations, e.g. in the Nambu and Jona-Lasinio (NJL) model [26. Recently, however, Geiger and Isgur presented a parameter-free analysis within a rather general framework consistent with the many empirical constraints (such as OZI rule), which shows that a complete set of strong strange mesonbaryon loops, computed in a model consistent with the OZI rule, gives (after delicate cancellations) only small observable $s \bar{s}$ effects. We therefore do not consider the $s \bar{s}$-effects from the second order in $\mathcal{L}_{I}$.

\section{Strangeness evaluation with a specified interaction $\mathcal{L}_{I}$}

The evaluation of Eq. (19) is in principle straightforward once one specifies two things. The first is the overall description of hadronic structure, which in practice amounts to choosing the model for the nucleon state $\left|N_{0}\right\rangle$ - for example, choosing some mean-field Hamiltonian such as $H_{0}$ in (12). The second is the choice of the interaction (we call it $\mathcal{L}_{I}$ ) which has the role to generate $q \bar{q}$ 


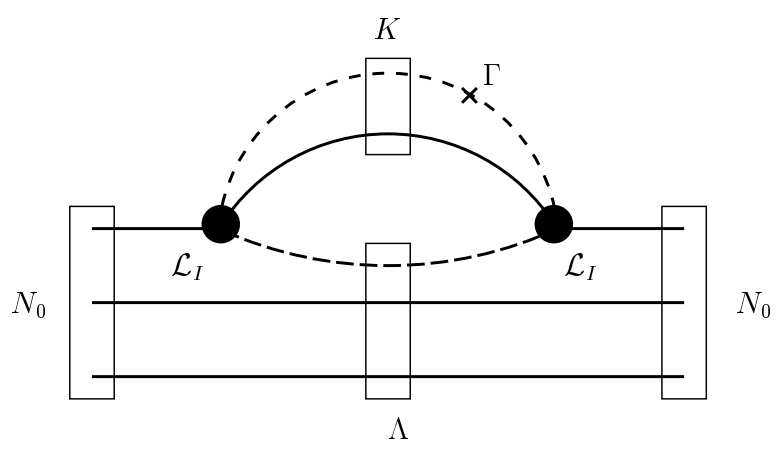

Figure 3: A response of the valence nucleon state $\left|N_{0}\right\rangle$ to a strangeness source at $\Gamma$ through two interactions $\mathcal{L}_{I}$. This type of contribution can be associated with the kaon-loop contribution to the nucleon strangeness (a possible $K \Lambda$ intermediate state is therefore indicated).

fluctuations on top of the valence nucleon state $\left|N_{0}\right\rangle$ (the eigenstate of $H_{0}$ ). Specifying $H_{0}$ also defines the model single-quark solutions, and we can use them as an appropriate wave-function basis to expand the quark fields $q(x)(q=u, d, s)$ in terms of creation $\left(\mathcal{U}_{K}^{\dagger}, \mathcal{D}_{K}^{\dagger}, \mathcal{S}_{K}^{\dagger}\right)$ and annihilation $\left(\mathcal{U}_{K}, \mathcal{D}_{K}, \mathcal{S}_{K}\right)$ operators of dressed quarks and antiquarks:

$$
q(x)=\sum_{K}\left(\mathcal{Q}_{K} q_{K}(\boldsymbol{x}) e^{-i \omega_{K} t}+\mathcal{Q}_{K}^{c^{\dagger}} q_{K}^{c}(\boldsymbol{x}) e^{i \omega_{K} t}\right) .
$$

Here, $q_{K}(\boldsymbol{r})$ denotes a model wave function of a quark of flavour $q$, where $K$ stands for the set of quantum numbers labelling a model quark state. (For example, in the next section we will choose to employ the MIT bag model. Then, $q_{K}(\boldsymbol{r})$ will be the solution for the quark in the $K$-th mode of the MIT bag.)

The field contractions in (19) lead to the sums over stationary modes of single quarks and antiquarks (such as the sums (27) and (28), evaluated in the next section), or, equivalently, to the bound-state propagators of these dressed model quarks. The sum over quark modes should naturally run up only to some typical hadronphysics low-energy cut-off $\Lambda \sim 0.6-1 \mathrm{GeV}$. This cutoff on quark energies is dictated by the fact that nonperturbative interactions between quarks operate at low energies, whereas they gradually weaken and go over to the perturbative regime at higher energies. (In the aforementioned study of $s \bar{s}$ effects of kaon loops [18], Geiger and Isgur have shown the importance of high-mass intermediate states in these loops. However, since these are hadronic, meson-baryon intermediate states, this does not contradict with cut-off such as $\Lambda \sim 1 \mathrm{GeV}$ on quark energies. Namely, the dominant portions of the results of Ref. [18 are accounted for by states lying below 3-3.5 $\mathrm{GeV}$. For comparison, our cut-off of $1.1 \mathrm{GeV}$ (see Table 1) imposed on the energies of one strange quark and one antiquark fluctuating on top of the valence nucleon state, corresponds to total energies up to $2 \Lambda+M_{N} \sim$
$3 \mathrm{GeV}$ as well. This leads us to believe that we have accounted for the majority of important degrees of freedom.) The cut-off values such as ours, are typical of calculations in models of low-energy QCD, e.g., the NJL model [26]. Obviously, we suppose here that the nucleon strangeness is the effect of low-energy non-perturbative QCD. Indeed, this brings us to the question what to use concretely for $\mathcal{L}_{I}$ in Eq. (19) in the explicit calculation of $\langle N|: \bar{s} \Gamma s:| N\rangle$.

The Lagrangian $\mathcal{L}_{I}$ can, of course, be any interaction that can produce fluctuating $s \bar{s}$ pairs, but the question is which interactions can be important in producing the strangeness of the nucleon. For example, perturbative QCD interactions probed in high-energy deep inelastic scattering and revealing the sea of $q \bar{q}$ pairs, including $s \bar{s}$, should be relatively unimportant in this regard 27, 28. A theoretical analysis [29] of the CCFR data [30] on strange quark distribution functions from neutrino-nucleon deep inelastic scattering seems to support this point of view. For example, it finds a very small upper bound on the strange radius of the nucleon $\left(\left|\left\langle r^{2}\right\rangle_{s}\right| \leq 0.005 \mathrm{fm}^{2}\right)$ 29]. The possibly enhanced nucleon strangeness is thus expected (see, e.g. [27]) as an effect of non-perturbative QCD, which, at low energies, around the nucleon mass scale, is certainly more important for hadronic structure than perturbative QCD, and can lead to $s \bar{s}$ pairs already at small momentum transfers, i.e. large distances. Non-perturbative QCD is after all responsible for precisely such effects as forming of a quark-antiquark condensate $\langle 0|\bar{q} q| 0\rangle(q=u, d, s)$ and a gluon condensate characterizing the non-perturbative QCD vacuum. Some investigators (see, e.g. [31], [3, 32], or, for comprehensive reviews, 33, 34, 35) have suggested that instantons are among the most important non-perturbative configurations of the gluon fields. By now it has been certainly well-established that the effective interaction between quarks resulting from the presence of instantons (let us call this interaction $\mathcal{L}_{\text {inst }}$ ) plays a very important role in the formation of hadron structure 33, 34, although it is not responsible for confinement [36, 37], as thought previously. (In the present approach, confinement must anyway be taken care of by the unperturbed Hamiltonian $H_{0}$.) In our opinion, this $\mathcal{L}_{\text {inst }}$ is therefore worth testing as an important candidate for the interactions $\mathcal{L}_{I}$ generating the strange nucleon matrix elements of some operators. A calculation [26] in the context of the NJL model seems to be an indication that $\mathcal{L}_{\text {inst }}$ is indeed the most important part of $\mathcal{L}_{I}$. The calculation in 26 found that large strange-pair components were present in the nucleon only if the instanton-induced interaction was included in low-energy dynamics.

Here we quote the vacuum-averaged version of the instanton-induced interaction $\mathcal{L}_{\text {inst }}$ derived by 4 in the instanton-liquid approach but transformed to the $x$ space. It is convenient to separate it into one-, two- and three-body pieces (Eq. (11)) $\mathcal{L}_{1}, \mathcal{L}_{2}$ and $\mathcal{L}_{3}$, respectively: 


$$
\begin{aligned}
& \mathcal{L}_{1}=-n\left(\frac{4 \pi^{2}}{3} \rho^{3}\right)\left\{\mathcal{F}_{u} \bar{u}_{R} u_{L}+(u \leftrightarrow d)+(u \leftrightarrow s)\right\} \\
& +(R \leftrightarrow L), \\
& \mathcal{L}_{2}=-n\left(\frac{4 \pi^{2}}{3} \rho^{3}\right)^{2}\left\{\mathcal { F } _ { u } \mathcal { F } _ { d } \left[\left(\bar{u}_{R} u_{L}\right)\left(\bar{d}_{R} d_{L}\right)\right.\right. \\
& \left.+\frac{3}{32}\left(\bar{u}_{R} \lambda^{a} u_{L} \bar{d}_{R} \lambda^{a} d_{L}-\frac{3}{4} \bar{u}_{R} \sigma_{\mu \nu} \lambda^{a} u_{L} \bar{d}_{L} \sigma^{\mu \nu} \lambda^{a} d_{L}\right)\right] \\
& +(u \leftrightarrow s)+(d \leftrightarrow s)\}+(R \leftrightarrow L), \\
& \mathcal{L}_{3}=-n\left(\frac{4 \pi^{2}}{3} \rho^{3}\right)^{3} \mathcal{F}_{u} \mathcal{F}_{d} \mathcal{F}_{s} \frac{1}{3 !} \frac{1}{N_{c}\left(N_{c}^{2}-1\right)} \\
& \quad \times \epsilon_{f_{1} f_{2} f_{3}} \epsilon_{g_{1} g_{2} g_{3}}\left\{\frac{2 N_{c}+1}{2 N_{c}+4}\left(\bar{q}_{R}^{f_{1}} q_{L}^{g_{1}}\right)\left(\bar{q}_{R}^{f_{2}} q_{L}^{g_{2}}\right)\left(\bar{q}_{R}^{f_{3}} q_{L}^{g_{3}}\right)\right. \\
& \left.+\frac{3}{8\left(N_{c}+2\right)}\left(\bar{q}_{R}^{f_{1}} q_{L}^{g_{1}}\right)\left(\bar{q}_{R}^{f_{2}} \sigma_{\mu \nu} q_{L}^{g_{2}}\right)\left(\bar{q}_{R}^{f_{3}} \sigma^{\mu \nu} q_{L}^{g_{3}}\right)\right\} .
\end{aligned}
$$

Here, $n$ is the instanton density and $\mathcal{F}_{f}$ 's are the characteristic factors (corresponding to inverse effective quark masses) composed of current light-quark masses $m_{f}(f=$ $u, d, s)$, average instanton size $\rho \simeq 1 / 3 \mathrm{fm}$ [38, 33, 32], and the quark condensate $\langle 0|\bar{q} q| 0\rangle$. For example, for the $u$ flavour, $\mathcal{F}_{u} \equiv\left[m_{u} \rho-\left(2 \pi^{2} / 3\right) \rho^{3}\langle 0|\bar{u} u| 0\rangle\right]^{-1}$, and analogously for the other flavours. The left (and right) projected components are defined in the usual way, $u_{L, R}=$ $\gamma_{\mp} u \equiv(1 / 2)\left(1 \mp \gamma_{5}\right) u$.

In the three-body interaction $\mathcal{L}_{3}$, the indices $f_{i}, g_{i}$ $(i=1,2,3)$ run over light flavours $u, d$ and $s$. For example, $g_{3}=d$ means $q_{L}^{g_{3}}=d_{L}$. Repeated indices are summed over. The interaction defined here by $\mathcal{L}_{1}, \mathcal{L}_{2}$ and $\mathcal{L}_{3}$ is actually the same as the well-known one of Shifman, Vainshtein and Zakharov (SVZ) 2], although the present three-body term (24) looks much simpler. In fact, Nowak 39] simply Fierzed away very complicated colour structures present in the SVZ interaction [2], reshuffling them to simple prefactors involving the number of quark colours $N_{c}$.

Although Nowak et al. derived this interaction in the random instanton liquid model (RILM) with the help of the mean-field, or quenched, approximation (where the collective coordinates of instantons and anti-instantons are randomly distributed, thus neglecting potentially important correlations) and in the long-wavelength limit, their version of the interaction induced by small instantons is still considered useful even in the most recent reviews of instanton physics 35. Ref. [- 4 took into account the delocalization of zero modes and long-wavelength properties (scales $>1 / 3 \mathrm{fm}$ ), arriving at the interaction basically corresponding to that of SVZ [2], apart from the effects of smearing over the average size $\rho$ of a small instanton, $\rho \simeq 1 / 3 \mathrm{fm} 38$, 3, 32]. In the limit of no smearing the SVZ instanton-induced interaction is obtained, that is, the interaction averaged over the small instanton volume is taken to be the local interaction (22)-24. In the long-wavelength limit, it should approximate well the intermediate-range $(\sim 1 / 3 \mathrm{fm})$ QCD effects, which are already of non-perturbative origin, but still not responsible for confinement appearing at still larger scales.

We also note that the average instanton size $\rho \simeq 1 / 3$ $\mathrm{fm}=(600 \mathrm{MeV})^{-1}$ is consistent with what we have said above about the typical hadronic cut-off scale $\Lambda \sim 0.6-1$ $\mathrm{GeV}$. Namely, the effective interaction $\mathcal{L}_{\text {inst }}$ cannot be operative at energies which would probe distances significantly smaller than the average size of these extended objects, instantons, which produce $\mathcal{L}_{\text {inst }}$.

Obviously, the two-body term is the one which, through Eqs. (19) and (20), yields the graph in Fig. 2 . The contribution to the nucleon strangeness due to the three-body interaction $\mathcal{L}_{3}$ is exemplified by the last loop in Fig. 1. Such graphs come about when contractions in (20) are performed with a strange bilinear in $\mathcal{L}_{3}$. The $\bar{s} s$ bilinear in the one-body term $\mathcal{L}_{1}$ can produce the strange quark loops disconnected from the valence quarks.

We should also comment on the consistency of using the instanton-induced interaction $\mathcal{L}_{\text {inst }}$ for $\mathcal{L}_{I}$ in Eq. (19), even when we view Eq. (19) as a purely perturbative result.

If we take the perturbative viewpoint, why is Eq. (19) applicable not only to parts of $\mathcal{L}_{I}$ which come from perturbative interactions such as the perturbative gluon exchange, but also to $\mathcal{L}_{\text {inst }}$ (22)-(24) which is of nonperturbative origin? The point is that the origin of $\mathcal{L}_{\text {inst }}$ is non-perturbative, i.e. these effective interactions between quarks are the consequence of non-perturbative gluon configurations - instantons. However, $\mathcal{L}_{\text {inst }}$ itself contains a small parameter, namely the instanton density $n$, and it is so small that a perturbative expansion in its powers is possible. Original estimates [38 where $n \approx 1.6 \cdot 10^{9} \mathrm{MeV}^{4}=1 \mathrm{fm}^{4}$ — have proved to be reliable as they have remained essentially unchanged [34] also in the more recent instanton-liquid calculations. It is useful to define a "dimensionless instanton density" $\tilde{n}$ by expressing it in units of the average instanton size $\rho, n \equiv \widetilde{n} \rho^{-4}$. The commonly accepted value is $\rho=1 / 600 \mathrm{MeV}^{-1} \simeq 1 / 3 \mathrm{fm} \mathrm{33,} \mathrm{32,} \mathrm{40].} \mathrm{Therefore,}$ $\widetilde{n} \simeq 12.4 \cdot 10^{-3} \simeq 1 / 81$, and this dimensionless parameter indicates that the probability of finding an instanton is small. $\widetilde{n}$ is obviously small enough to be used as the parameter of the perturbative expansion. We should also keep in mind that this is the instanton density in the true, non-perturbative QCD vacuum, while in some circumstances the appropriate $n$ can be even smaller. Notably, Ref. 41] has found that in the MIT bag model enlarged with the instanton-induced interaction (22)-24), which is used in the next section for the first evaluations of the nucleon strangeness using formula (19), the instanton density is very strongly depleted with respect to the true $\mathrm{QCD}$ vacuum. 
Of course, this depletion relative to the instanton density in the true QCD vacuum, is just the way to express the small probability of penetration of the instanton liquid from the RILM vacuum (modeling the true QCD vacuum supposedly outside the bag) into parts of the volume inside the otherwise perturbative bag. If one wants, this can be visualized as occasional penetration into the bag (with a small probability), of drops of the instanton liquid. It is important to note that it is not a different kind of liquid, with different properties, but that it must be the same liquid - namely the one giving rise to the interaction (22)-(24). For that reason, we use in the instanton-induced interaction $\mathcal{L}_{I}(22)-(24)$, as usual, the empirical value of the condensate $\langle 0|\bar{q} q| 0\rangle$.

The non-vanishing (albeit small) probability for penetration of the droplets of instanton liquid from the true non-perturbative "instanton vacuum" of QCD into the "perturbative" MIT bag interior explains why, in the case of the MIT bag, we should be concerned with the strangeness coming from the one-body term $\mathcal{L}_{1}$. It is true that this term does not involve any interaction with valence quarks and one would thus expect that it is already included in the vacuum contribution. Nevertheless, recall that in the MIT bag model, Donoghue and Nappi [5] obtained the strangeness of the "perturbative" MIT bag by subtracting the (negative) non-perturbative vacuum contribution, since everything is measured with respect to the true non-perturbative QCD vacuum as the referent, "zero" level. However, if there is a non-vanishing probability for penetration of droplets of the random instanton liquid vacuum of QCD, it means that the difference of the bag interior with respect to the true nonperturbative QCD vacuum was not so large. Hence, the over-subtracted strangeness should be put back in, and this is the reason for the one-body term $\mathcal{L}_{1}$ contributing to our $s \bar{s}$-pairs on top of the vacuum.

\section{Instanton-induced strangeness in the MIT bag model}

We now turn to the actual calculation of strange nucleon matrix elements in a specific model, and with the instanton-induced interaction $\mathcal{L}_{\text {inst }}$ given by Eqs. (22, 24). For definiteness, we quote the results for the proton - the neutron case is quite similar. Using Eq. (19), we can write the proton-strangeness matrix element as

$$
\begin{aligned}
& \langle N|: \bar{s} \Gamma s:| N\rangle=i \int_{-\infty}^{\infty} d t^{\prime}\left\langle N_{0}\right| \hat{T}: \int d^{3} x \\
& \times \bar{s}\left(\boldsymbol{x}, t_{0}\right) \Gamma s\left(\boldsymbol{x}, t_{0}\right):: \int d^{3} y \mathcal{L}_{\text {inst }}\left(\boldsymbol{y}, t^{\prime}\right):\left|N_{0}\right\rangle
\end{aligned}
$$

where we have kept only the first term in the perturbation series over low instanton density. We have treated each of the three parts of $\mathcal{L}_{\text {inst }}$ (11) separately. The onebody interaction $\mathcal{L}_{1}(22)$ is the simplest of all. Since no
Table 1: Strange-quark energy levels $\omega_{n \kappa}$, which can be excited by the instanton interaction.

\begin{tabular}{|c|c|c|}
\hline$n$ & $\kappa$ & $\omega_{n \kappa} / \mathrm{MeV}$ \\
\hline 0 & -1 & 514.0 \\
0 & -2 & 726.7 \\
0 & 1 & 797.4 \\
1 & -1 & 1104.9 \\
\hline
\end{tabular}

valence quarks take part in this interaction, the only relevant part of $\mathcal{L}_{1}$ is

$$
-n\left(\frac{4 \pi}{3} \rho^{3}\right) \mathcal{F}_{s}\left[\bar{s}_{R}\left(\boldsymbol{y}, t^{\prime}\right) s_{L}\left(\boldsymbol{y}, t^{\prime}\right)+\bar{s}_{L}\left(\boldsymbol{y}, t^{\prime}\right) s_{R}\left(\boldsymbol{y}, t^{\prime}\right)\right]
$$

Expanding the strange-quark fields $s$ like in Eq. (21) and contracting them leads to the following contribution of the one-body interaction $\mathcal{L}_{1}(22)$ to the matrix element (25):

$$
\begin{aligned}
& \langle N|: \bar{s} \Gamma s:| N\rangle_{\mathcal{L}_{1}}=4 \pi^{2} n \rho^{3} \mathcal{F}_{s} \sum_{K, L} \frac{1}{\omega_{K}+\omega_{L}} \\
\times & \left\{\int d^{3} x \bar{s}_{K}(\boldsymbol{x}) \Gamma s_{L}^{c}(\boldsymbol{x}) \int d^{3} y \bar{s}_{L}^{c}(\boldsymbol{y}) s_{K}(\boldsymbol{y})+\left(s \leftrightarrow s^{c}\right)\right\} .
\end{aligned}
$$

We now choose the MIT bag as our concrete model for the nucleon円. Therefore, the wave functions $q_{K}(\boldsymbol{r})$ $(q=u, d, s)$ denote the MIT bag model solutions? and $K$ stands for the set $\left\{n, \kappa, j_{3}\right\}$, where $n$ is the radial excitation number and the quantum number $\kappa$ is determined by the total and orbital angular momenta $j$ and $l$, respectively. $\omega_{K}$ is the energy of the quark in the bag state $K$. With all this, the one-body contribution to the nucleon strangeness is completely specified.

The sum over $K=\left\{n, \kappa, j_{3}\right\}$ and $L=\left\{n^{\prime}, \kappa^{\prime}, j_{3}^{\prime}\right\}$ goes up to the state with $n=1, \kappa=-1$ (corresponding to the cut-off of about $1.1 \mathrm{GeV}$ ), encompassing four lowest-lying strange quark states displayed in Table 1 . The expression for the contribution of the two-body interaction $\mathcal{L}_{2}(23)$ is obtained in the same way as Eq. (27). However, it is somewhat more complicated, involving also valence quark wave functions. Luckily, the terms

\footnotetext{
1 The problem that the MIT bag model has with the breaking of the chiral symmetry on the bag boundary, is cured in various versions of the chiral bag model containing the pion fields outside the bag with quarks, complete with appropriate boundary conditions. In the models where the bag radius is as large as in the ordinary MIT bag [42], the pion field outside is so weak that it does not perturb significantly the quark sector where our $\mathcal{L}_{I}$ acts, and cannot influence the strangeness much. Therefore, the results obtained in such a chirally invariant but more complicated model, should not be very different from those obtained in the simple MIT bag model, so that in the next section, we stick to the latter for concreteness and simplicity.

${ }^{2}$ We follow the conventions of Ref. 43 for the MIT bag wave functions. See also our more complete account 44, where we give technicalities in detail.
} 
with $\sigma^{\mu \nu}$ cancel out, leaving us with the proton matrix element

$$
\begin{gathered}
\langle N|: \bar{s} \Gamma s:| N\rangle_{\mathcal{L}_{2}}=\frac{16}{3} \pi^{4} n \rho^{6} \mathcal{F}_{q} \mathcal{F}_{s} \sum_{K, L, \pm} \frac{1}{\omega_{K}+\omega_{L}} \\
\times\left\{\int d^{3} x \bar{s}_{K}(\boldsymbol{x}) \Gamma s_{L}^{c}(\boldsymbol{x}) \int d^{3} y \bar{s}_{L}^{c}(\boldsymbol{y}) \gamma_{ \pm} s_{K}(\boldsymbol{y})\right. \\
\times\left[2 \bar{q}_{0,-1, \frac{1}{2}}(\boldsymbol{y}) \gamma_{ \pm} q_{0,-1, \frac{1}{2}}(\boldsymbol{y})\right. \\
\left.+\bar{q}_{0,-1,-\frac{1}{2}}(\boldsymbol{y}) \gamma_{ \pm} q_{0,-1,-\frac{1}{2}}(\boldsymbol{y})\right] \\
+\int d^{3} x \bar{s}_{K}^{c}(\boldsymbol{x}) \Gamma s_{L}(\boldsymbol{x}) \int d^{3} y \bar{s}_{L}(\boldsymbol{y}) \gamma_{ \pm} s_{K}^{c}(\boldsymbol{y}) \\
\times\left[2 \bar{q}_{0,-1, \frac{1}{2}}(\boldsymbol{y}) \gamma_{ \pm} q_{0,-1, \frac{1}{2}}(\boldsymbol{y})\right. \\
\left.\left.+\bar{q}_{0,-1,-\frac{1}{2}}(\boldsymbol{y}) \gamma_{ \pm} q_{0,-1,-\frac{1}{2}}(\boldsymbol{y})\right]\right\} .(28)
\end{gathered}
$$

Here, $q_{0,-1, \pm \frac{1}{2}}(\boldsymbol{y})$ is the wave function for the ground state of the valence quark in the bag, which we take to be the same for $u$ and $d$ quarks.

Going now to the three-body interaction $\mathcal{L}_{3}(24)$, expressions become extremely long and complicated, so we do not write them down here. As seen below, it turns out that this contribution is much smaller than the preceding two, anyway.

After focusing on the scalar $(\bar{s} s)$ and pseudoscalar $\left(\bar{s} \gamma_{5} s\right)$ strangeness as the channels preferred by the QCDvacuum fluctuations [1], we have also checked the vector $\left(\bar{s} \gamma_{\mu} s\right)$ and the axial-vector $\left(\bar{s} \gamma_{\mu} \gamma_{5} s\right)$ channels.

The calculation of the contribution of the two-body $\mathcal{L}_{2}$ and the three-body $\mathcal{L}_{3}$ instanton interactions is tedious and in the manipulation of all these formulae we have relied heavily on Mathematica package 45] for symbolic computer calculations.

To illustrate how our calculations in the MIT bag model have been performed and in which way such a model choice influences our results, we briefly sketch the calculation with the one-body part $\mathcal{L}_{1}$ interaction.

\subsection{Scalar and pseudoscalar strangeness}

Let us first consider the scalar strange current density $\bar{s} s$ inside the proton. The expression for the matrix element can be written as

$$
\left\langle N\left(p^{\prime}\right)\left|\bar{s} s e^{-i \boldsymbol{q} \cdot \boldsymbol{x}}\right| N(p)\right\rangle=A_{s}\left(q^{2}\right) \bar{u}_{N}\left(p^{\prime}\right) u_{N}(p),
$$

where $q^{2}=\left(p-p^{\prime}\right)^{2}$, and $u_{N}$ 's are nucleon spinors. $A_{s}\left(q^{2}\right)$ is the scalar form factor accounting at $q^{2}=0$ for the scalar strangeness of the proton.

Calculations inside the bag model can be performed by making the substitution $\Gamma=1$ and inserting the appropriate quark and antiquark wave functions in (27).
By a simple calculation one can show that the surviving combination is the one with $\kappa=-1, \kappa^{\prime}=1$ and $\kappa=1$, $\kappa^{\prime}=-1$, and (27) reduces to

$$
\begin{aligned}
& \langle N|: \bar{s} s:| N\rangle_{\mathcal{L}_{1}}=4 \pi^{2} n \rho^{3} \mathcal{F}_{s} \sum_{n=0}^{1} \frac{4}{\omega_{n,-1}+\omega_{0,1}} \\
& \times\left[N_{-1}\left(x_{n,-1}\right) N_{1}\left(x_{0,1}\right) \int r^{2} d r\right. \\
& \times W_{+}(n,-1) W_{-}(0,1) j_{0}\left(x_{n,-1} \frac{r}{R}\right) j_{0}\left(x_{0,1} \frac{r}{R}\right) \\
& \left.\quad+W_{-}(n,-1) W_{+}(0,1) j_{1}\left(x_{n,-1} \frac{r}{R}\right) j_{1}\left(x_{0,1} \frac{r}{R}\right)\right]^{2}
\end{aligned}
$$

The normalizations $N_{ \pm 1}\left(x_{n, \pm 1}\right)$ and the $W_{ \pm}$-factors, related to the quark wave functions, are given in Refs. 443] and 44 .

The above equation represents the contribution to the strange scalar form factor $A_{s}\left(q^{2}=0\right)$ coming from the one-body interaction. The remaining contributions from the $\mathcal{L}_{2}$ and $\mathcal{L}_{3}$ instanton interactions can be calculated similarly and the results are

$$
\begin{aligned}
& \langle N|: \bar{s} s:| N\rangle_{\mathcal{L}_{1}}=0.035 \\
& \langle N|: \bar{s} s:| N\rangle_{\mathcal{L}_{2}}=0.023 \\
& \langle N|: \bar{s} s:| N\rangle_{\mathcal{L}_{3}}=2.9 \cdot 10^{-4} .
\end{aligned}
$$

Summing them up gives

$$
A_{s}(0)_{\mathcal{L}_{\text {inst }}}=0.058 .
$$

The evaluation of space-integrals has been performed numerically using the following values: the bag radius $R=1 / 197.3 \mathrm{MeV}^{-1} \approx 1 \mathrm{fm}$, the average instanton size $\rho=1 / 600 \mathrm{MeV}^{-1}$ and the instanton density $n=2.66 \cdot 10^{7}$ $\mathrm{MeV}^{4}$, which is the depleted instanton density in the MIT bag as found in [11. Moreover, we have taken the strange quark mass $m_{s}=200 \mathrm{MeV}$ and the valence quark mass $m_{u}=m_{d} \equiv m_{q}=8 \mathrm{MeV}$. The quark condensate that follows from the Gell-Mann-Oakes-Renner relation for these quark masses and the empirical meson masses is $\langle 0|\bar{q} q| 0\rangle \approx(-200 \mathrm{MeV})^{3}$.

The pseudoscalar strange form factor $B_{s}$ is defined as

$$
\left\langle N\left(p^{\prime}\right)\left|\bar{s} \gamma_{5} s e^{-i \boldsymbol{q} \cdot \boldsymbol{x}}\right| N(p)\right\rangle=B_{s}\left(q^{2}\right) \bar{u}_{N}\left(p^{\prime}\right) \gamma_{5} u_{N}(p) .
$$

For the pseudoscalar strange current $\bar{s} \gamma_{5} s$, Eq. (27) gives the vanishing one-body contribution

$$
\left\langle N\left|: \bar{s} \gamma_{5} s:\right| N\right\rangle_{\mathcal{L}_{1}}=0 .
$$

Analogously, we obtain the vanishing result for the other two instanton interactions, i.e. $\left\langle N\left|: \bar{s} \gamma_{5} s:\right| N\right\rangle_{\mathcal{L}_{\text {inst }}}=0$. We thus obtain,

$$
B_{s}(0)_{\mathcal{L}_{\text {inst }}}=0,
$$

as the vanishing total instanton contribution to the pseudoscalar form factor. 


\subsection{Vector and axial-vector strangeness}

In Eq. (5) the vector strangeness has been displayed in terms of the Dirac $\left(F_{1}^{s}\right)$ and the Pauli $\left(F_{2}^{s}\right)$ form factors. For comparison with experimental data, the (strange) Sachs form factors $G_{E}^{s}$ (electric) and $G_{M}^{s}$ (magnetic) are widely used:

$$
\begin{aligned}
G_{E}^{s}\left(q^{2}\right) & =F_{1}^{s}\left(q^{2}\right)+\frac{q^{2}}{4 M_{N}^{2}} F_{2}^{s}\left(q^{2}\right), \\
G_{M}^{s}\left(q^{2}\right) & =F_{1}^{s}\left(q^{2}\right)+F_{2}^{s}\left(q^{2}\right) .
\end{aligned}
$$

By taking the non-relativistic nucleon spinor

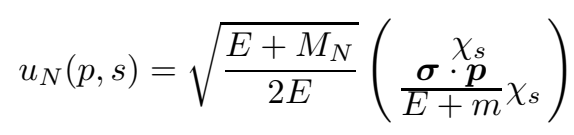

in the Breit frame defined by

$$
\begin{aligned}
q^{\mu} & =\left(q^{0}, \boldsymbol{q}\right)=\left(0, \boldsymbol{q}_{B}\right), \\
\boldsymbol{p} & =\frac{\boldsymbol{q}_{B}}{2}, \boldsymbol{p}^{\prime}=-\frac{\boldsymbol{q}_{B}}{2},
\end{aligned}
$$

the components of the vector current take the form

$$
\begin{aligned}
& \left\langle N\left(p^{\prime}, s^{\prime}\right)\left|V_{0}^{s}\right| N(p, s)\right\rangle=\frac{m}{E} \chi_{s^{\prime}}^{\dagger} \chi_{s} G_{E}^{s}\left(-\boldsymbol{q}_{B}^{2}\right), \\
& \left\langle N\left(p^{\prime}, s^{\prime}\right)\left|\boldsymbol{V}^{s}\right| N(p, s)\right\rangle=\frac{1}{2 E} \chi_{s^{\prime}}^{\dagger} i\left(\boldsymbol{\sigma} \times \boldsymbol{q}_{B}\right) \chi_{s} G_{M}^{s}\left(-\boldsymbol{q}_{B}^{2}\right)
\end{aligned}
$$

In order to calculate the contribution of the instanton-induced vector strange current inside the MIT bag, we have to identify the form factors in (42) with the Fourier-transformed vector current within the bag:

$$
\begin{aligned}
& \left\langle N\left(p^{\prime}\right)\left|: V_{\mu}^{s}:\right| N(p)\right\rangle_{\mathcal{L}_{\text {inst }}} \\
& =\left\langle N\left(p^{\prime}\right)\left|: \int d^{3} r e^{-i \boldsymbol{q}_{B} \cdot \boldsymbol{r}} \bar{s}(\boldsymbol{r}) \gamma_{\mu} s(\boldsymbol{r}):\right| N(p)\right\rangle_{\mathcal{L}_{\text {inst }}}
\end{aligned}
$$

using the static limit $q \rightarrow 0$. The check with the $V_{0}^{s}$ component of the vector current gives zero, i.e. $G_{E}^{s}\left(q^{2}=\right.$ $0)_{\text {inst }}=0$, as it should be.

A similar calculation for the space components $\boldsymbol{V}^{s}$ shows a non-trivial cancellation among the contributions of quarks in the loop with different spin orientations producing the total result

$$
G_{M}^{s}(0)_{\mathcal{L}_{\text {inst }}}=0 .
$$

This implies the vanishing strange magnetic moment

$$
\mu_{s}=F_{2}^{s}(0)=0,
$$

which is compatible with the recent measurements at MIT/Bates 46] and even more recent ones at TJNAF (JLab) 477.

is

Eq. (9) then implies that the baryomagnetic moment

$$
\mu_{p}^{(0)}=1+\kappa_{p}+\kappa_{n}=0.88 \mathrm{n} . \mathrm{m} .
$$

The estimation of the axial-vector strangeness can be done along the same lines. The form-factor decomposition, assuming the $G$-parity symmetry of the strong interactions, has the form

$$
\begin{aligned}
& \left\langle N\left(p^{\prime}\right)\left|\bar{s} \gamma_{\mu} \gamma_{5} s\right| N(p)\right\rangle \\
& =\bar{u}_{N}\left(p^{\prime}\right)\left(\gamma_{\mu} \gamma_{5} G_{1}^{s}\left(q^{2}\right)+\frac{q_{\mu}}{2 M_{N}} \gamma_{5} G_{2}^{s}\left(q^{2}\right)\right) \bar{u}_{N}(p)(.47)
\end{aligned}
$$

The instanton contribution to such a matrix element can be calculated as

$$
\begin{aligned}
& \left\langle N\left(p^{\prime}\right)\left|: A_{\mu}^{s}:\right| N(p)\right\rangle_{\mathcal{L}_{\text {inst }}} \\
& =\left\langle N\left(p^{\prime}\right)\left|: \int d^{3} r e^{-i \boldsymbol{q}_{B} \cdot \boldsymbol{r}} \bar{s}(\boldsymbol{r}) \gamma_{\mu} \gamma_{5} s(\boldsymbol{r}):\right| N(p)\right\rangle_{\mathcal{L}_{\mathrm{ins}}(48)}
\end{aligned}
$$

and should be compared with the axial form factors defined in the Breit frame as

$$
\left\langle N\left(p^{\prime}, s^{\prime}\right)\left|\boldsymbol{A}^{s}\right| N(p, s)\right\rangle=G_{A}^{s}(0) \chi_{s^{\prime}}^{\dagger} \boldsymbol{\sigma} \chi_{s} .
$$

Again, it turns out that the axial-vector strangeness induced by the instanton interaction is vanishing,

$$
G_{A}^{s}(0)_{\mathcal{L}_{\text {inst }}}=0 .
$$

\section{Discussion and conclusions}

This paper deals with strange quarks at very small momentum transfers $Q^{2}$, as opposed to the high values of $Q^{2}$, where such non-valence components of nucleon are undisputable, and also treatable using more standard methods of perturbative QCD and parton models. The original MIT bag model 48, 49, 50] represents a suitable starting point in predicting the low-energy properties of low-mass hadrons. In this model, $R_{\text {bag }}$ imitates the separations $R_{\text {confining }} \sim 1 \mathrm{fm}$ at which confinement effects are important, corresponding to the confining scale $\Lambda_{Q C D} \simeq$ 100 to $300 \mathrm{MeV}$. Short-distance effects are supposedly taken care of by the perturbative one-gluon exchange.

However, in order to account for the effects at intermediate distances, i.e. at momentum scales $Q \sim \Lambda_{\chi S B} \simeq$ $0.6-1 \mathrm{GeV}$, the effective interaction (11), (22)-(24), induced by the liquid of small instantons (of the average size $\rho=1 / 3 \mathrm{fm}$ ) appears appropriate. Of course, the effects of the instanton-induced interactions are not included in Donoghue and Nappi's [5] naive bag-model relation (3) for the scalar nucleon strangeness, and the relative importance of this naive strangeness and the instanton effects is precisely what interests us here.

An advantage of formula (19) is that, at least in principle, it treats the scalar, pseudoscalar, vector, axial, tensor or pseudotensor nucleon strangeness in a unified manner - one just has to specify what $\Gamma$ is. Within a chosen nucleon model, the evaluation of $(19)$ would proceed in essentially the same way for each $\Gamma$, except for technical details. 
In the scalar case $(\Gamma=1)$, the naive bag-model strangeness (3) is actually rather large for standard values of parameters. For our values, given at the end of subsection 4.1 , it is

$$
A_{s}^{\mathrm{Nbag}} \equiv-\langle 0|\bar{q} q| 0\rangle V_{\mathrm{bag}}=4.36,
$$

which is much larger than the instanton-induced contribution (34), and dominates the summed strangeness

$$
A_{s} \equiv A_{s}^{\mathrm{Nbag}}+A_{s}(0)_{\mathcal{L}_{\text {inst }}}=4.42 .
$$

Owing to using a somewhat smaller value of the quark condensate, Donoghue and Nappi [5] obtained 3.6 for this naive strangeness, which is still rather large. $A_{s}^{\text {Nbag }}$ depends very strongly on the model size parameter $R_{\text {bag }}$ since $V_{\text {bag }}=R_{\text {bag }}^{3} 4 \pi / 3$. For example, $A_{s}^{\text {Nbag }}$ would decrease by a factor of 2 if $R_{\text {bag }}=0.8 \mathrm{fm}$, a nucleon size which may be more acceptable, as the standard MIT bag value of $1 \mathrm{fm}$ seems too large (e.g., see 51). However, since the model dependence on the bag radius is similar for other presently interesting matrix elements, the model dependence largely cancels out when one forms ratios. In particular, the instanton-induced contribution (34) remains small in comparison with the naive nucleon bag strangeness:

$$
\frac{A_{s}^{\mathrm{Nbag}}}{A_{s}(0)_{\mathcal{L}_{\text {inst }}}} \sim 75
$$

for reasonable variations of the radius parameter.

Obviously, the contribution due to the difference in the condensate with respect to the true, nonperturbative QCD vacuum, dominates the strangeness in the nucleon bag. Admittedly, the instanton-induced contribution of this size would be obtained in the calculation of Eq. (34) if, inside the MIT bag, the non-depleted instanton density $n=1.6 \cdot 10^{9} \mathrm{MeV}^{4}$ were used. However, we consider this merely as a consistency check, and not as an alternative description of strangeness in the MIT bag. This is because using the instanton density appropriate to the non-perturbative QCD vacuum containing the large quark condensate, would imply that the non-perturbative $\mathrm{QCD}$ vacuum and the quark condensate were assumed not only outside, but also inside the bag. This would indeed enable $A_{s}(0)_{\mathcal{L}_{\text {inst }}}$ to replace $A_{s}^{\text {Nbag }}$ in full, but would also make the MIT bag description inconsistent [41].

The scalar strangeness is special because of non-vanishing scalar $\bar{q} q$ condensates of the QCD vacuum, which makes it more natural that it is larger than vector, axial or other kinds of strangeness. This is especially clear in our approach applied to the MIT bag model. In this model, the scalar strangeness comes mostly from the difference of the scalar $\bar{q} q$ condensates in the true $\mathrm{QCD}$ vacuum and their absence in the perturbative vacuum inside the cavity [5], while only the relatively small remainder in the present paper comes from the response of the valence ground state to the strangeness-sensitive probe.
However, such a response is all that exists in the case of the pseudoscalar, vector, axial, etc., nucleon strangeness, since there are no pseudoscalar, vector, axial, etc., QCDvacuum condensates either inside or outside the cavity. Since such responses tend to be much smaller than the non-perturbative vacuum contributions, significant differences in magnitude between the scalar and other kinds of strangeness are very natural in our approach. In fact, in the present case of the MIT bag model, we find the vanishing first-order contribution to the vector strangeness. The vanishing first-order contributions are also found for the pseudoscalar and axial strangeness of the nucleon.

Thus, our results confirm the conjecture of Ref. [1] for the case of the scalar strangeness.

This makes understandable why the results on the "non-scalar" strange quantities, such as the strangeness nucleon magnetic form factor [10, 11, 12, 13, 14, 16, 17, 19, 20, 21, 22 or the strangeness electric mean-square radius [10, 13, 14, 15, 16, 17, 18, 19, 20, 21, 22], vary so much, even by the sign, from one model to another: the "non-scalar" strange quantities should all be rather small, and artifacts of various models very easily put it on either side of the zero.

Our results are also consistent with the most recent measurements of the strange vector form factors at low momentum transfer, $Q^{2} \lesssim 1 \mathrm{GeV}$. The experimental strange magnetic form factor of the nucleon at $Q^{2}=0.1$ $(\mathrm{GeV} / \mathrm{c})^{2}, G_{M}^{s}=0.23 \pm 0.37 \pm 0.15 \pm 0.19$ n.m., obtained at MIT/Bates [46] is consistent with the absence of strange quarks, but the error bars are large. However, the results and conclusions of our approach, that channels other than the scalar one should not be appreciably affected by strange quarks, seems to get support especially from the most recent and very precise TJNAF (JLab) measurement [47] yielding the small strange vector form factors at $Q^{2}=0.48(\mathrm{GeV} / \mathrm{c})^{2}, G_{E}^{s}+0.39 G_{M}^{s}=$ $0.023 \pm 0.034 \pm 0.022 \pm 0.026$ n.m. Furthermore, HAPPEX collaboration 44] plans to improve the accuracy of this result by a factor of two in 1999. Nevertheless, its small central value, consistent with zero, and small errors, already exclude some of the more generous predictions [10, 21] for the strangeness (but not [52, 14] for example).

\section{Acknowledgement}

D. K. and I. P. thank I. Zahed for getting them started in this problem, and for many illuminating discussions. D. K., K. K. and I. P. acknowledge the partial support of the EU contract CI1*-CT910893 (HSMU), and the hospitality of the Physics Department of the Bielefeld University.

\section{References}

[1] A. R. Zhitnitsky, Phys. Rev. D55 (1997) 3006 
[2] M. A. Shifman, A. I. Vainshtein and V. I. Zakharov, Nucl. Phys. B163 (1980) 46

[3] D. I. Diakonov and V. Y. Petrov, Nucl. Phys. B245 (1984) 259

[4] M. A. Nowak, J. J. M. Verbaarschot and I. Zahed, Nucl. Phys. B324 (1989) 1

[5] J. F. Donoghue and C. R. Nappi, Phys. Lett. 168B (1986) 105

[6] V. M. Khatsimovskii, I. B. Khriplovich and A. R. Zhitnitsky, Z. Phys. C36 (1987) 455

[7] J. Ellis and M. Karliner, Phys. Lett. B341 (1995) 397

[8] J. Ashman et al., Phys. Lett. 206B (1988) 364

[9] J. Ashman et al., Nucl. Phys. B328 (1989) 1

[10] R. L. Jaffe, Phys. Lett. B229 (1989) 275

[11] D. Klabučar and I. Picek, Phys. Lett. B231 (1989) 170

[12] D. Klabučar and I. Picek, Nucl. Phys. A514 (1990) 689

[13] N. W. Park, J. Schechter and H. Weigel, Phys. Rev. D43 (1991) 869

[14] M. J. Musolf and M. Burkardt, Z. Phys. C61 (1994) 433

[15] H. Forkel et al., Phys. Rev. C50 (1994) 3108

[16] S.-T. Hong, B.-Y. Park and D.-P. Min, Phys. Lett. B414 (1997) 229

[17] S. T. Hong and B. Y. Park, Nucl. Phys. A561 (1993) 525

[18] P. Geiger and N. Isgur, Phys. Rev. D55 (1997) 299

[19] D. B. Leinweber, Phys. Rev. D53 (1996) 5115

[20] D. B. Leinweber, Nucl. Phys. A585 (1995) 341c

[21] H. W. Hammer, U.-G. Meissner and D. Drechsel, Phys. Lett. B367 (1996) 323

[22] C. V. Christov et al., Prog. Part. Nucl. Phys. 37 (1996) 1

[23] L. A. Ahrens et al., Phys. Rev. D35 (1987) 785

[24] R. P. Feynman, Phys. Rev. 56 (1939) 340

[25] H. Hellmann, Einführung in die Quantenchemie (Deuticke, Leipzig, 1937)

[26] K. Steininger and W. Weise, Phys. Lett. B329 (1994) 169
[27] B. L. Ioffe and M. Karliner, Phys. Lett. B247 (1990) 387

[28] R. Decker, M. Nowakowski and U. Wiedner, Fortschr. Phys. 41 (1993) 87

[29] X. Ji and J. Tang, Phys. Lett. B362 (1995) 182

[30] A. O. Bazarko et al., Z. Phys. C65 (1995) 189

[31] B. V. Geshkenbein and B. L. Ioffe, Nucl. Phys. B166 (1980) 340

[32] D. I. Diakonov and V. Y. Petrov, Nucl. Phys. B272 (1986) 457

[33] E. V. Shuryak, The QCD Vacuum, Hadrons and the Superdense Matter (World Scientific, Singapore, 1988)

[34] E. V. Shuryak, hep-ph/9503427

[35] T. Schafer and E. V. Shuryak, Rev. Mod. Phys. 70 (1998) 323

[36] J. Greensite, Nucl. Phys. B249 (1985) 263

[37] Y. A. Simonov, Sov. J. Nucl. Phys. 50 (1989) 310, [Yad. Fiz. 50 (1989) 500]

[38] E. V. Shuryak, Nucl. Phys. B203 (1982) 93

[39] M. A. Nowak, Acta Phys. Polon. B22 (1991) 697

[40] E. V. Shuryak, Nucl. Phys. B302 (1988) 599

[41] D. Klabučar, Phys. Rev. D49 (1994) 1506

[42] R. L. Jaffe, in Lectures at the 1979 Summer school, Ettore Majorana, edited by A. Zichichi, vol. 17 (Plenum Press, New York, 1982) MIT preprint CTP 814 (1979)

[43] L. J. Carson, R. J. Oakes and C. R. Willcox, Phys. Rev. D33 (1986) 1356

[44] D. Klabučar et al., in preparation

[45] S. Wolfram, Mathematica: A System for Doing Mathematics by Computer (Addison-Wesley, 1988)

[46] B. Mueller et al., Phys. Rev. Lett. 78 (1997) 3824

[47] K. A. Aniol et al., Phys. Rev. Lett. 82 (1999) 1096

[48] A. Chodos et al., Phys. Rev. D9 (1974) 3471

[49] A. Chodos et al., Phys. Rev. D10 (1974) 2599

[50] T. DeGrand et al., Phys. Rev. D12 (1975) 2060

[51] G. Brown et al., Z. Phys. A331 (1988) 139

[52] H. Weigel et al., Phys. Lett. B353 (1995) 20 